\title{
Emergence of vancomycin-resistant Enterococcus at a tertiary paediatric hospital in South Africa
}

\author{
H Lochan, ${ }^{1,2 *}$ MB ChB, DCH, FCPaed (SA), Cert ID (SA) Paed; C Moodley, ${ }^{3,4 *}$ PhD (Mol Cell Biol); D Rip, ${ }^{3,4}$ PhD (Biotechnol); \\ C Bamford, ${ }^{3,4}$ MB ChB, DCH (SA), MPhil (Mat Child Health), FCPath (Microbiol), MMed (Med Microbiol); M Hendricks, ${ }^{2,5}$ MB ChB, DIP \\ PEC (SA), DCH (SA), FCPaed (SA), CMO Paed (SA); A Davidson, ${ }^{2,5}$ MB ChB, DCH (SA), FCPaed (SA), CMO Paed (SA), MPhil; \\ B Eley, ${ }^{1,2} \mathrm{MB} \mathrm{ChB}, \mathrm{FCPaed}(\mathrm{SA}), \mathrm{BSc}$ Hons \\ ${ }^{1}$ Paediatric Infectious Diseases Unit, Red Cross War Memorial Children's Hospital, Cape Town, South Africa \\ ${ }^{2}$ Department of Paediatrics and Child Health, School of Child and Adolescent Health, Faculty of Health Sciences, University of Cape Town, \\ South Africa \\ ${ }^{3}$ Division of Medical Microbiology, Department of Pathology, Faculty of Health Sciences, University of Cape Town, South Africa \\ ${ }^{4}$ National Health Laboratory Service, Groote Schuur Hospital, Cape Town, South Africa \\ ${ }^{5}$ Haematology/Oncology Service, Red Cross War Memorial Children's Hospital, Cape Town, South Africa \\ ${ }^{*}$ These authors contributed equally to the work (joint first authors).
}

Corresponding author: B Eley (brian.eley@uct.ac.za)

\begin{abstract}
Background. During 2013, the haematology/oncology unit at a tertiary level paediatric hospital in South Africa experienced the emergence of infection with vancomycin-resistant Enterococcus (VRE).

Objective. To describe the clinical and molecular aspects of the cases identified.

Methods. VRE isolates identified from blood culture specimens processed at the National Health Laboratory Service were screened for the presence of the vancomycin resistance genes van $\mathrm{A}, \mathrm{B}$ and $\mathrm{C} 1,2$ and 3 . Further characterisation of these isolates was carried out using pulsedfield gel electrophoresis (PFGE) and multilocus sequence typing (MLST). Clinical records of infected patients were reviewed to identify possible risk factors, while surveillance with rectal swabs was performed to identify VRE-colonised patients.

Results. Four patients with haematological malignancies were identified with VRE bloodstream infections. Patients were immunocompromised at the time of the bloodstream infection (BSI), with receipt of vancomycin prior to VRE-BSI, and infections were treated with linezolid. Colonisation with VRE was found in 8 of 55 patients screened. Infected and colonised patients were isolated in the unit during their admission and strict contact precaution infection control practices were instituted. The vanA gene was identified in all of the isolates but one. PFGE and MLST results showed a degree of genetic relatedness between certain isolates obtained from rectal swab and blood culture samples, suggesting possible patient-to-patient transmission or persistence of the isolates in the unit.

Conclusion. Strict infection control practices are necessary to prevent infection and transmission of resistant organisms among vulnerable patients.
\end{abstract}

S Afr Med J 2016;106(6):562-566. DOI:10.7196/SAMJ.2016.v106i6.10858

Multidrug-resistant organisms are an increasing threat to healthcare worldwide. Treatment options for these so-called 'superbugs' are limited, with very few new antimicrobials currently in development.

One such organism is vancomycin-resistant Enterococcus (VRE). This organism has been detected worldwide in hospitalised adult and paediatric patients since the first description in $1986 .{ }^{[1]}$ Infection and/or colonisation with VRE has been described in patients who are potentially immunosuppressed, especially those with malignancies, solid organ transplant recipients, those with chronic renal disease requiring haemodialysis, neonates and those requiring long-term ventilation. ${ }^{[2,3]}$

Resistance to vancomycin occurs in E. faecium and E. faecalis species primarily owing to the $\operatorname{van} \mathrm{A}$ and $\operatorname{van} \mathrm{B}$ resistance genes. The first clinical reports of VRE in South African (SA) adults appeared in 1997 and there is currently a paucity of SA paediatric studies describing the incidence of VRE or outbreaks of this organism. ${ }^{[4]}$

We report on the first identification of VRE infections in the paediatric oncology ward at a tertiary-level paediatric hospital in Cape Town, SA, over two time periods in 2013.

\section{Methods}

\section{Identification}

The first two VRE organisms causing bloodstream infections (VREBSI) were isolated from blood culture specimens of two patients, 2 days apart, in February 2013. Seven and 9 months later, in September and November 2013, respectively, VRE was once again isolated from blood culture specimens in an additional two patients. Following the two time periods, patient hospital records were accessed to identify possible risk factors associated with acquiring VRE-BSI in this setting. Patient records were anonymised and de-identified prior to analysis. Routine screening is not carried out for drug-resistant organisms on admission to the hospital.

\section{Setting}

The haematology/oncology unit at Red Cross War Memorial Children's Hospital (RCWMCH) in Cape Town, SA, is a 17-bed unit with a high-care unit and isolation cubicles. There is a daily outpatient service and a day ward in the area adjoining the main ward. It serves as a referral centre for children with haematological and solid organ malignancies from other local or distant hospitals in the country. Children are admitted from home or long-term 
care facilities. The unit has approximately 2000 admissions, with an additional 5500 outpatient visits, per year

\section{Surveillance}

Following identification of the first two VRE infections, collaboration between the paediatric oncologists, microbiologists, paediatric infectious diseases physicians, infection control nursing staff and ward nursing staff was instituted to limit further spread by improving infection prevention and control measures. Active surveillance for rectal colonisation with VRE of patients in the ward and discharged patients who had prior contact with the index cases was carried out by means of culture and sensitivity testing using rectal swabs or stool samples. If rectal swabs were negative for VRE at initial screening, a repeat was taken at 1 - 2-weekly intervals until three consecutive negative swabs were documented. ${ }^{[5]}$

\section{Infection control}

Infected patients were isolated in single cubicles until discharged. Contact precautions during patient interaction were instituted for patients, family and staff members. Colonised patients were cohorted until discharged. Medical records were marked to ensure isolation, and re-screening by rectal swab occurred during subsequent admissions. Staff and parents were reeducated regarding correct handwashing technique, use of alcohol hand sanitiser and contact precautions.

\section{Microbiological methods}

All microbiological and molecular analyses were conducted by the National Health Laboratory Service at Groote Schuur Hospital and the Division of Medical Microbiology, University of Cape Town (UCT), SA. Surveillance specimens were plated directly onto selective media (colistin nalidixic blood agar impregnated with a $30 \mu \mathrm{g}$ vancomycin disc) and incubated for up to 48 hours. Identification and susceptibility testing of colonies growing within the inhibition zone close to the vancomycin disc were performed using the Vitek 2 GP and AST-P603 cards respectively, with interpretation according to contemporary Clinical Laboratory Standards Institute criteria. ${ }^{[6]}$ Vancomycin minimum inhibitory concentrations (MICs) were determined using the Etest method. ${ }^{[6]}$ MICs $\geq 32 \mu \mathrm{g} / \mathrm{mL}$ were considered resistant.

Isolates were characterised genotypically with the Hain Genotype Enterococcus line probe assay according to the manufacturer's instructions, or in-house polymerase chain reaction (PCR) assay as described below.

\section{In-house PCR assay for genotyping enterococci}

All vancomycin-resistant isolates were screened for the presence of the vancomycin resistance genes $\operatorname{van} \mathrm{A}, \mathrm{B}$ and C1, 2, 3 with DNA purified using the ZR Fungal/Bacterial DNA MiniPrep kit (Zymo Research, USA) according to the manufacturer's instructions. ${ }^{[7]} \mathrm{PCR}$ reactions $(25 \mu \mathrm{L})$ were carried out using GoTaq Flexi (Promega, USA) with an annealing temperature of $60^{\circ} \mathrm{C}$, for 35 cycles, in a GeneAmp 9700 thermocycler. PCR amplicons were visualised by agarose gel electrophoresis and ethidium bromide staining. Amplicons were confirmed with DNA sequence analysis (Inqaba Biotech, SA) and BLASTN (http://blast.ncbi.nlm. nih.gov/Blast) comparisons. ${ }^{[8]}$

\section{Pulsed-field gel electrophoresis}

Isolates of interest were analysed using pulsed-field gel electrophoresis (PFGE), as described by the Centers for Disease Control and Prevention, with the following changes: cultures were grown on BHI medium, at $37^{\circ} \mathrm{C}$, aerobically overnight, and cell densities were standardised to an $\mathrm{OD}_{610 \mathrm{~mm}} \approx 1.0$, using a spectrophotometer (BioDrop, UK) ${ }^{[9]}$ Cells were embedded in a 1.0\% SeaKem Gold agarose gel (Lonza, Switzerland) and were digested for 2 hours at $25^{\circ} \mathrm{C}$ using $\mathrm{SmaI}$ (Thermo Scientific, USA) restriction endonuclease and ApaI as a secondary enzyme ( 2 hours at $37^{\circ} \mathrm{C}$ ). Digested plugs were electrophoresed through a $1.0 \%$ SeaKem Gold agarose gel (Lonza, Switzerland) in 0.5\% TBE buffer $\left(14^{\circ} \mathrm{C}\right.$ ), using the Gene Navigator (Amersham Biosciences, UK) at $200 \mathrm{~V}$, with a ramped switching time of $3.5-23.5$ seconds over 18 hours. The resultant gel was visualised using ethidium bromide staining, and the gel image analysed and dendrogram generated using Gel Compare II (BioNumerics v.6.6, Applied-Maths, USA), with an optimisation of 1.0 and a band tolerance of 1.5. The dendrogram was constructed using the pairwise average from the SmaI and ApaI results, with the UPGMA (unweighted pair group method with arithmetical mean) method and branch quality was calculated using the cophenetic correlation. E. faecalis ATCC 51299 was used as a control and for normalisation.

\section{Multilocus sequence typing}

Cellular DNA was extracted from selected isolates and PCR was carried out as described above using the conditions described by Homan et al. ${ }^{[10]}$ Amplicons were sequenced bidirectionally (Inqaba

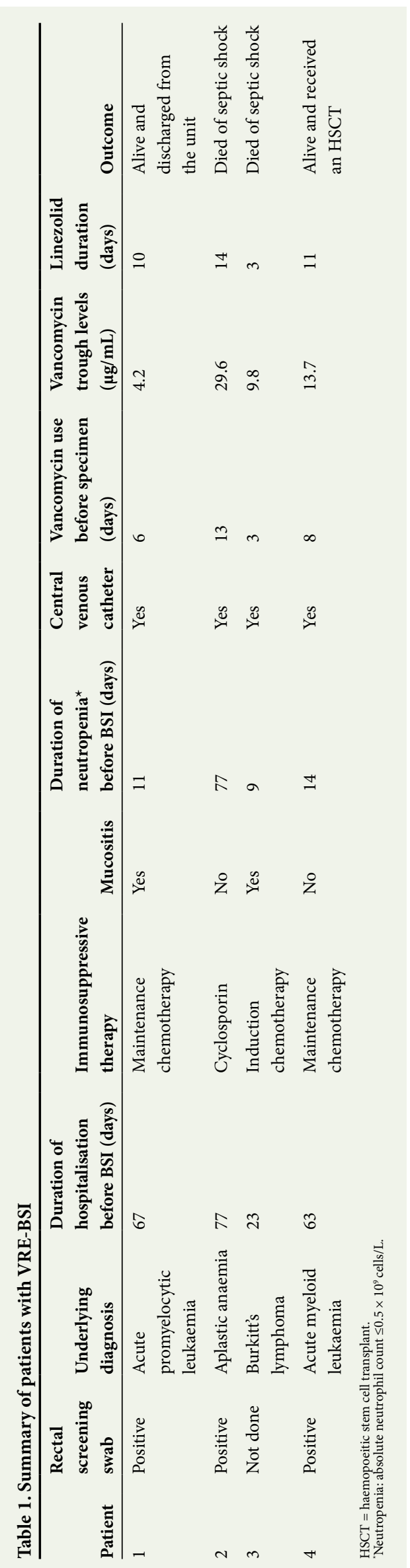


Biotech, SA) and the consensus sequences uploaded and typed at using an E. faecium multilocus sequence typing (MLST) database. ${ }^{[1]}$

\section{Ethical considerations}

Ethics approval to perform this study was obtained from the Human Research Ethics Committee, UCT (HREC Ref. No. 022/2015).

\section{Results}

\section{Description of the infection}

The ward had 100\% occupancy during the months of VRE isolation. All four BSI patients were long-term residents in the unit at the time of the infections and were therefore classified as hospital-acquired infections. Patients 2 and 4 were transferred from a referral hospital outside Cape Town, where VRE had previously been isolated. Neither of these patients was screened prior to their transfer to RCWMCH. The clinical features and underlying risk factors at the time of BSI were similar in all four identified patients (Table 1). Three of the infected patients cultured vancomycin-sensitive E. faecium (VSE) isolates from blood in the week preceding the VRE-BSI. All four were treated with vancomycin in the week prior to the the VRE-BSI, and three had suboptimal vancomycin trough drug levels $(\leq 15 \mu \mathrm{g} /$ $\mathrm{mL}$ ). Patient 2 had renal impairment which resulted in the increased trough levels of vancomycin. Central venous catheters were removed from all infected patients after detection of the VRE-BSI. Infected patients were treated with intravenous linezolid. Patients 2 and 3 had protracted periods of illness prior to the BSI and demised from a new Gram-negative BSI and typhilitis following the VRE-BSI, respectively.

\section{Surveillance}

Two of 29 (6.9\%) patients who were residing in the ward or recently discharged were screened (as identified above) by rectal swab for VRE during February and March 2013 and a further 6 of 26 (23.1\%) patients screened between September and October 2013 were positive for VRE. Patient 4 screened positive on rectal swab and subsequently progressed to develop a VRE-BSI a week later. Although the overall number of patients colonised with VRE was 14.5\% (8/55), surveillance swabbing was not routinely performed between March and September, and the true prevalence was therefore not established. There were, however, no cases of clinical disease due to VRE documented between March and September 2013. Environmental screening for VRE was not conducted in the unit.

\section{Microbiology}

All but one isolate obtained (surveillance and infection) was identified as E. faecium, using the Vitek 2 system. The additional isolate obtained on rectal swab was identified as E. faecalis based on vancomycin sensitivity testing. Vancomycin MICs on Etest were $\geq 256 \mu \mathrm{g} / \mathrm{mL}$ for the four BSI isolates, confirming high-level resistance of E. faecium to vancomycin. ${ }^{[8]}$

\section{Molecular testing}

PCR analysis detected the vanA gene in all of the VRE isolates obtained from infected patients and all but one of the VRE screening isolates. This isolate tested positive for the $\operatorname{vanB}$ gene. PFGE analysis of these isolates showed that there was genetic diversity among the isolates, which was not indicative of an outbreak. There was, however, a high level of genetic relatedness ( $>85 \%$ confidence interval) between some of the strains tested (Fig. 1). The E. faecalis isolate obtained during the February - March surveillance screening did not undergo any further molecular typing, even though the van $\mathrm{A}$ gene was detected on PCR.

Patients 1 and 2, who were considered to be the index cases of a suspected outbreak, had isolates which shared $92.5 \%$ homology, indicating a strong possibility of patient-to-patient transmission of the same clone. This result was unusual since the patient 2 isolate in question (No. 13) was obtained from a rectal swab and the isolate obtained from the blood culture of Patient 2 (No. 3) did not show strong homology with each other.

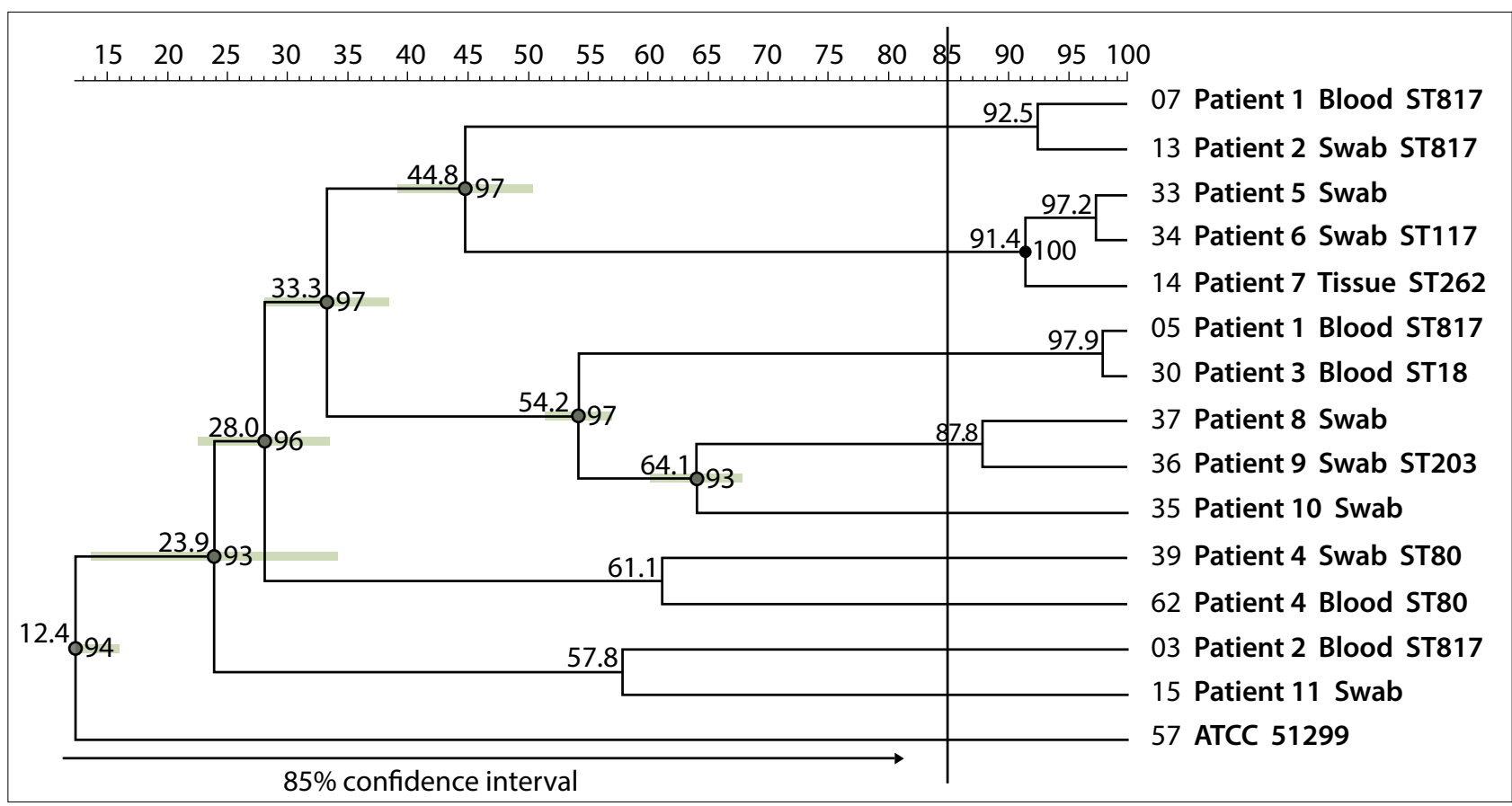

Fig. 1. Dendrogram of isolates obtained during investigation. Shaded bars indicate branch error flags and unshaded values indicate the levels of relatedness. Patients 1 - 4 represent the patients with VRE-BSIs. Patients 5, 6 and $8-11$ indicate the patients colonised with VRE (patient 11 in February and March, and patients 5, 6, 8, 9 and 10 in September and October; patient 7 was not a haematology/oncology patient). The specimen types and MLST sequence types are indicated in the last two columns, respectively. 
The second isolate from patient 1 (No. 5), obtained from a blood culture, shared $97.2 \%$ homology with the blood culture isolate from patient 3 (No. 30). Isolate No. 30 was faintly positive for the vanA gene on PCR and susceptible to vancomycin. Previous isolates from this patient produced strong positive PCR results and high levels of vancomycin resistance. None of the resistant isolates from patient 3 showed significant homology to the sensitive isolate or to any of the other strains tested (data not shown). The two isolates obtained from blood in patient 1 (Nos 5 and 7), however, shared very little genetic homology. These data indicated the possibility of a rapid rate of strain variation, even within the same patient, in this setting. The two isolates from patient 4 (Nos 39 and 62) shared only $61.1 \%$ homology with each other and very low homology with any of the other strains tested.

There were five screening strains, in two clusters, that shared high genetic homology with each other. Cluster 1 (91.4\% homology) comprised patients 5, 6 and 7 and cluster 2 (87.8\% homology) comprised patients 8 and 9. Patient 7 (No. 14) in cluster 1 was PCRpositive for the van $\mathrm{B}$ gene, but still shared high homology with the other two isolates, which were both vanA-positive. This patient had no prior contact with the haematology/oncology unit. The isolate was obtained from autopsy of the lung tissue and was probably a contaminant and not a comorbidity. Cluster 2 comprised two isolates that were obtained from different patients on the same day. These results indicated the possibility of spread within the hospital.

MLST analysis revealed that the four isolates from patients 1 and 2 (Nos 3, 5, 7 and 13) all shared the same sequence type (ST817). Even though these isolates do not share significant homology in the dendrogram, it strengthens the assumption that these two patients probably had patient-to-patient transmission of the same clone that may have changed over time. The two isolates from patient 4 (Nos 39 and 62 ) that shared $61.1 \%$ homology also shared the same sequence type (ST80). They are single-locus variants of ST817, with a single polymorphism in the $a d k$ gene $(189 \mathrm{G}>\mathrm{A})$.

\section{Infection control}

A multidisciplinary approach was instituted to prevent further spread of the organism within the oncology unit. Infection control staff assisted with ensuring that infected patients were isolated and all ward staff members were educated about contact precautions. Colonised patients were cohorted in the multi-bed cubicles for the duration of their hospital stay. Discharged patients were identified to determine who required screening on their next visit.

The ward was closed off to all new admissions until all colonised or infected patients were discharged. Once all colonised or infected patients were discharged, the ward was comprehensively cleaned.

Following the first two cases of VRE-BSI, antibiotic stewardship ward rounds were instituted in the oncology unit, to improve antibiotic management including optimisation of vancomycin dosing. All patients who required transfer from other hospitals to the oncology unit at RCWMCH, where VRE had previously been documented, were screened for VRE by rectal swab prior to transfer.

\section{Discussion}

This is the first documented report of VRE infection in children in SA. A prevalence study in SA found $10.9 \%$ (20/184) of screening rectal swabs in at-risk patients to be positive for enterococal isolates. ${ }^{[12]}$ This study also showed clonal spread of the $\operatorname{van} \mathrm{A}$ and $\operatorname{van} \mathrm{B}$ genotypes in different hospitals, as well as persistence of the van A carrying strain over time. The vanA genotype has been commonly isolated in previous outbreak settings. ${ }^{[2,3]}$

Even though the PFGE results indicated overall genetic diversity between the isolates analysed, there was a degree of genetic relatedness between specific blood and rectal swab samples on both PFGE and MLST analysis. These results may suggest possible patient-to-patient transfer of VRE or persistence of the organism in the haematology/ oncology unit between the two time periods described. Patient 4 was infected by a molecularly distinct, unrelated isolate, possibly before transfer to RCWMCH at a referral hospital where VRE had previously been isolated. The identified sequence type (ST80) along with ST203 (No. 36) and ST18 (No. 30) have previously been assigned to clonal complexes (CC) $\mathrm{CC} 117, \mathrm{CC} 78$, and $\mathrm{CC} 17$, respectively, which are known to form part of the $\mathrm{CC} 17$ meroclone. The CC17 meroclone has been described as highly diverse with documented worldwide spread. ${ }^{[13]}$ These data again highlight the high levels of strain variation observed in this setting.

The purpose of screening is to identify carriers during outbreaks, and in non-outbreak settings to prevent transmission, especially to vulnerable patient groups. ${ }^{[14]}$ Active screening outside outbreak settings can be a costly exercise and especially difficult in resourceconstrained health systems. Screening in children has revealed low rates of colonisation. Active screening of paediatric patients found that $3.4 \%$ (41/1 211) were colonised with VRE, of whom 39/41 (95.1\%) had an underlying chronic disease. ${ }^{[15]}$

Risk factors for acquiring a BSI or colonisation with this highly resistant organism have been investigated. There are few paediatric studies regarding the risk factors for acquiring VRE. Acquisition of VRE has been associated with previous treatment with broad-spectrum antimicrobials, especially third-generation cepholosporins, and the carbapenems, as well as immunosuppression with antineoplastic chemotherapy, haemotology/oncology and renal patients, increased length of stay in hospital, and a younger age in children. ${ }^{[13]}$ Progression from VRE colonisation to VRE-BSI in patients was associated with admission from a long-term care facility, infection of an additional body site other than blood and receipt of vancomycin. ${ }^{[16]}$ In contrast, Pentima et al. ${ }^{[17]}$ found that VRE-BSI was independent of patient vancomycin exposure. Risk factors in our patient cohort are similar to those described. All the patients with VRE-BSI had an underlying haematological condition requiring immunosuppressive therapy for treatment and received vancomycin for treating presumed Gram-positive infections. Central venous catheters were present in all four infected patients and, although not found to be a risk factor in the studies mentioned, could represent a possible clinical source for BSI. The colonised patients, although not clinically ill at the time, also had similar risk factors: all were exposed to vancomycin at some period during their illness and had underlying immunosuppression in the period preceding the screening. As mentioned, only one of the colonised patients progressed to developing bacteraemia with VRE.

Treatment for this resistant organism is limited to the use of linezolid and daptomycin. In SA, daptomycin is difficult to access in the public health system. Linezolid, an oxazolidinone antibacterial agent, has proven to be effective in the treatment of the glycopeptideresistant Enterococcus, as well as methicillin-resistant Staphylococcus aureus and penicillin-resistant Streptococcus pneumoniae in children, and was used to treat the infected patients in this study. ${ }^{[18]}$

\section{Study limitations}

The limitations of this study include lack of a case-control group to conclusively identify risk factors associated with VRE acquisition in our patients, and an insufficient sample size to ascertain whether an outbreak had occurred and to determine the definitive phylogenetic relationships of the VRE strains present in this setting. Owing to financial constraints, no active surveillance was possible after October 2013, so whether or not further transmission occurred and 
the duration of colonisation with VRE were not established. However, no further VRE cases were reported in the oncology unit or the hospital after November 2013.

\section{Conclusion}

In conclusion, effective infection control practices are important to limit or prevent transmission of multidrug-resistant pathogens among vulnerable hospitalised populations. Furthermore, the emergence of glycopeptide-resistant enterococci has emphasised the importance of antibiotic stewardship in the haematology/oncology unit and the institution overall.

Conflicts/disclosures. Parts of this work have been previously presented as a poster at the 16th International Congress on Infectious Diseases (ICID) in 2014 and published in the conference preceedings in the International Journal of Infectious Dieases (2014), as well as a poster presentation at the 9th World Congress of the World Society for Pediatric Infectious Diseases (WSPID) in 2015.

Authors' contributions. HL and CM participated in the drafting of the manuscript. BE conceived the study and helped in the drafting of the manuscript. CM and DR carried out the molecular typing of all the isolates. All authors read and approved the final manuscript.

. Leclercq R, Derlot E, Duval J, Courvalin P. Plasmid-mediated resistance to vancomycin and teicoplani in Enterococcus faecium. N Engl J Med 1988;319(3):157-161.

2. Iosifdis E, Karakoula K, Protonotariou E, et al. Polyclonal outbreak of vancomycin-resistant Enterococcus faecium in a paediatric oncology department. J Pediatr Hematol Oncol 2012;34(7):511516. DOI:10.1016/j.ajic.2013.02.005
3. Singh J, Esparza S, Patterson M, Vogel K, Patel B, Gornick W. Vancomycin-resistant Enteroococcus in paediatric oncology patients: Balancing infection prevention and family-centered care. I Pediatr Hematol Oncol 2013;35(3):227-231. DOI:10.1097/MPH.0b013e318257a6ca

4. Budavari SM, Saunders GL, Liebowitz LD, Khoosal M, Crewe-Brown, HH. Emergence of vancomycinresistant enterococci in South Africa. S Afr Med J 1997;87(11):1557.

Siegel ID, Rhinehart E, Jackson M, Chiarello L. Management of Multidrug-Resistant Organisms in Healthcare Settings. Atlanta, Ga, USA: Centers for Disease Control and Prevention, 2006.

Clinical and Laboratory Standards Institute. Performance Standards for Antimicrobial Susceptibility

Testing. Wayne, Penn., USA: CLSI, 2013.
. Bell JM, Paton JC, Turnidge J. Emergence of vancomycin-resistant enterococci in Australia: Phenotypic 7. Bell JM, Paton JC, Turnidge J. Emergence of vancomycin-resistant enterococci in
and genotypic characteristics of isolates. J Clin Microbiol 1998;36(8):2187-2190.

8. Altschul SF, Gish W, Miller W, Lipman DJ. Basic local alignment search tool. J Mol Biol 1990;215(3):403410.

9. Centers for Disease Control. Unified Pulsed-Field Gel Electrophoresis (PGFE). Protocol for Gram Positive Bacteria. Atlanta, Ga, USA: CDC, 2012. http://www.cdc.gov/hai/pdfs/labSettings/Unified PGFE_Protocol.pdf (accessed 9 December 2014).

10. Homan WL, Tribe D, Poznanski S, et al. Multilocus sequence typing scheme for Enterococcus faecium. J Clin Microbiol 2002;40(6):1963-1971. DOI:10.1128/jcm.40.6.1963-1971.2002

11. Enterococcus faecium MLST Databases. http://pubmlst.org/efaecium (accessed 25 September 2015).

12. Von Gottberg A, van Nierop W, Duse A, et al. Epidemiology of glycopeptide-resistant enterococci colonizing high risk patients in hospitals in Johannesburg, Republic of South Africa. J Clin Microbiol 2000;38(2):905-909.

13. Ergani-Ozcan A, Naas T, Baysan BO, et al. Nosocomial outbreak of vancomycin-resistant Enterococcus faecium in a paediatric unit at a Turkish university hospital. J Antimicrob Chemother 2008;61(5):10331039. DOI:10.1093/jac/dkno66

14. Humphreys $\mathrm{H}$. Controlling the spread of vancomycin-resistant enterococci: Is active screening worthwhile? J Hosp Infect 2014;88(4):191-198. DOI:10.1016/j.jhin.2014.09.002

15. Weddle G, Jackson MA, Selvarangan R. Utility of a focused vancomycin-resistant enterococci screenin protocol to identify colonization in hospitalized children. Am J Infect Control 2012;40(9):891-892. DOI:10.1016/j.ajic.2011.12.005

16. Olivier CN, Blake RK, Steed LL, Salgado C. Risk of vancomycin-resistant Enterococcus (VRE) bloodstream infection among patients colonized with VRE. Infect Control Hosp Epidemio 2008;29(5):404-409. DOI:10.1086/587647

17. Pentima MCD, Chan S, Briody C, Power M, Hossain J. Driving forces of vancomycin-resistant $E$. faecium and $E$ faecalis blood-stream infections in children. Antimicrob Resist Infect Control 2014;3(1):29-33. DOI:10.1186/2047-2994-3-29

18. Dotis J, Iosifidis E, Ioannidou M, Roilides E. Linezolid use in paediatrics: A critical review. Int J Infect Dis 2010;14(8):e638-e648. DOI:10.1016/j.jiji.2009.10.002

Accepted 11 April 2016 